Leukemia (2019) 33:2531-2535

https://doi.org/10.1038/s41375-019-0475-z

Chronic lymphocytic leukemia

\title{
Phase 1 first-in-human trial of the anti-CD37 antibody BI 836826 in relapsed/refractory chronic lymphocytic leukemia
}

\author{
Stephan Stilgenbauer ${ }^{1} \cdot$ Thérèse Aurran Schleinitz $^{2} \cdot$ Barbara Eichhorst $^{3} \cdot$ Fabian Lang $^{4} \cdot$ Fritz Offner $^{5}$. \\ Jean-François Rossi ${ }^{6}$. Wilfried Schroyens ${ }^{7}$ - Eric Van Den Neste ${ }^{8}$ - Loïc Ysebaert ${ }^{9}$ - Ute von Wangenheim ${ }^{10}$. \\ Uta Ursula Kress ${ }^{10}$. Petra Blum ${ }^{10}$. Thorsten Zenz ${ }^{11,12}$
}

Received: 22 November 2018 / Revised: 22 March 2019 / Accepted: 25 March 2019 / Published online: 14 May 2019

(c) The Author(s) 2019. This article is published with open access

\section{To the Editor:}

Chronic lymphocytic leukemia (CLL) remains incurable for most patients, requiring repeated therapy [1]. The tetraspanin CD37 is predominantly expressed on mature B cells, with low-level expression on $\mathrm{T}$ cells, granulocytes, and monocytes, and is widely expressed in mature B-cell malignancies, including CLL [2]. Upon cross-ligation, CD37 can function as a cell death receptor in CLL cells [3]. Thus, CD37 is an attractive target for CLL.

BI 836826 is a chimeric mouse-human monoclonal antibody that targets human CD37 with a dual mode of action that induces antibody-dependent cell-mediated cytotoxicity and has intrinsic proapoptotic activity [4]. BI 836826 has demonstrated stringent antitumor effects in

Presented in part at the 20th Congress of the European Hematology Association; June 11-14, 2015; Vienna, Austria.

Supplementary information The online version of this article (https:// doi.org/10.1038/s41375-019-0475-z) contains supplementary material, which is available to authorized users.

Stephan Stilgenbauer

Stephan.Stilgenbauer@uniklinik-ulm.de

1 Department of Internal Medicine III, Ulm University, 89081 Ulm, Germany

2 Department of Hematology, Institute Paoli-Calmettes, Marseille, France

3 Department I of Internal Medicine, Center for Integrated Oncology Cologne Bonn, University of Cologne, Cologne, Germany

4 Department of Hematology/Oncology, Goethe University Hospital, Frankfurt am Main, Germany

5 Universitair Ziekenhuis Gent, Gent, Belgium

6 Département d'Hématologie Clinique, CHU Montpellier, Université Montpellier, 80 Avenue Augustin Fliche, 34295 preclinical cell-based assays and xenograft models, with superior activity versus rituximab [4]. These data warrant evaluation of BI 836826 in CLL and other mature B-cell malignancies. Here, we report a phase 1 first-in-human, open-label, dose-escalation study to evaluate the maximum tolerated dose (MTD), safety and efficacy of BI 836826 in relapsed/refractory CLL (NCT01296932).

Eligible patients had relapsed/refractory CLL according to the International Workshop on Chronic Lymphocytic Leukemia (IWCLL) definition [5], and had received $\geq 2$ prior treatment regimens (Supplementary Patients and Methods). The study was conducted in accordance with the Declaration of Helsinki and Good Clinical Practice guidelines, and the protocol approved by the relevant institutional review boards. Patients provided written informed consent.

BI 836826 was administered at a starting dose of $1 \mathrm{mg}$ (Supplementary Patients and Methods; Suppl. Fig. 1). Dose escalation followed a modified $3+3$ design based on protocol-defined dose-limiting toxicities (DLTs; Supplementary Materials and Methods) during the first treatment cycle. Primary endpoints were MTD and number of patients with DLTs during cycle 1. MTD was defined as the highest

\section{Montpellier, France}

7 Antwerp University Hospital and University of Antwerp, Antwerp, Belgium

8 Department of Hematology, Cliniques Universitaires Saint-Luc \& Université Catholique de Louvain, B-1200 Brussels, Belgium

9 IUCT-Oncopole, Toulouse, France

10 Boehringer Ingelheim Pharma GmbH \& Co KG, Biberach an der Riss, Germany

11 Department of Medicine V, University Hospital Heidelberg, Department of Molecular Therapy in Haematology \& Oncology, NCT/DKFZ, Heidelberg, Germany

12 Department of Medical Oncology and Hematology, University Hospital Zürich and University of Zürich, Zürich, Switzerland 
dose of BI 836826 for which the incidence of DLT was no more than $1 / 6$ patients during cycle 1 . Patients received up to four treatment cycles, with additional cycles permitted in the case of clinical benefit. An expansion cohort was planned but did not occur because the trial was terminated due to the lack of recruitment after new drugs (idelelisib/ ibrutinib) received marketing approval.

Adverse events (AEs) were graded according to the NCICTCAE (National Cancer Institute-Common Terminology Criteria for Adverse Events) (version 4.0) and collected up to 42 days after the last dose of BI 836826 (on-treatment period). Efficacy endpoints included (amongst others): best percentage change in peripheral lymphocyte count from baseline; number of patients with improved hemoglobin levels, platelet counts and neutrophil counts (Supplementary Patients and Methods); and best overall response by investigator assessment according to IWCLL criteria [5]. Blood samples for pharmacokinetic analysis were taken pre dose and at prespecified timepoints post dose (Supplementary Patients and Methods). Pharmacodynamic assessments were undertaken centrally and included assessment of circulating B lymphocyte antigen (CD19+) and CD19+/CD37+ cell counts (Supplementary Patients and Methods). Statistical analyses were descriptive and exploratory; no formal statistical tests were performed. Primary endpoints were based on the MTD evaluable set; the treated set was used for all other analyses.

Forty-five patients were screened across ten sites in three countries from November 2011 to October 2015. Thirtyseven patients were treated in eight cohorts: $1 \mathrm{mg}(n=3)$, $3 \mathrm{mg}(n=3), 9 \mathrm{mg}(n=6), 25 \mathrm{mg}(n=6), 50 \mathrm{mg}(n=3)$, $100 \mathrm{mg} \quad(n=3), 200 \mathrm{mg} \quad(n=6), 400 \mathrm{mg}(n=3)$, and $800 \mathrm{mg}(n=4)$. Median age (range) was 68 (44-80) years, $70 \%$ were male, and $65 \%$ had a del(17p) and/or TP53 mutation (Suppl. Table 1). Patients received a median of 7 cycles (range $1-68) ; 13$ patients $(35.1 \%)$ received 8 cycles and five $(13.5 \%)$ received $>8$ cycles. At the time of primary analysis, 36 patients had discontinued treatment due to: progressive disease $(n=10)$, DLT $(n=2)$, patient refusal $(n=5)$, other $\mathrm{AE}(n=7)$, or other reasons $(n=12)$. Two patients (400 mg; $9 \mathrm{mg}$ escalating to $25 \mathrm{mg}$ after 16 cycles) derived prolonged clinical benefit and remained on treatment for nearly 3 years (70 and 68 cycles, respectively).

Two of 35 evaluable patients experienced a DLT in cycle 1; grade 3 hypophosphatemia (200 mg cohort) and grade 4 neutropenia lasting $>7$ days (800 mg cohort; Suppl. Table 2). Based on on-treatment DLTs and AEs leading to discontinuation, $800 \mathrm{mg}$ was not considered tolerable. No DLTs were observed in patients receiving $400 \mathrm{mg}$. Doses between 400 and $800 \mathrm{mg}$ were not explored as the trial was discontinued. A posthoc Bayesian logistic regression model indicated that $600 \mathrm{mg}$ might be acceptable for further development.
Table 1 Overall summary of AEs, and treatment-related AEs occurring in $>10 \%$ of patients

\begin{tabular}{|c|c|c|}
\hline & $\begin{array}{l}\text { Any grade } \\
n(\%)\end{array}$ & $\begin{array}{l}\text { Grade } 3 \text { or } 4 \\
n(\%)\end{array}$ \\
\hline Any AE & $37(100)$ & $31(83.8)$ \\
\hline Treatment-related AE & 35 (94.6) & $27(73.0)$ \\
\hline Any SAE & $18(48.6)$ & $13(35.1)$ \\
\hline Treatment-related SAE & $12(32.4)$ & $8(21.6)$ \\
\hline $\begin{array}{l}\text { AE leading to treatment } \\
\text { discontinuation }\end{array}$ & $10(27.0)$ & 7 (18.9) \\
\hline \multicolumn{3}{|l|}{$\begin{array}{l}\text { Most common treatment- } \\
\text { related AEs }\end{array}$} \\
\hline Infusion-related reaction & $26(70.3)$ & $3(8.1)$ \\
\hline Chills & $22(59.5)$ & $0(0.0)$ \\
\hline Pyrexia & $18(48.6)$ & $1(2.7)$ \\
\hline Neutropenia & $17(45.9)$ & $16(43.2)$ \\
\hline Thrombocytopenia & $12(32.4)$ & $9(24.3)$ \\
\hline Anemia & $10(27.0)$ & $6(16.2)$ \\
\hline Dyspnea & 8 (21.6) & $2(5.4)$ \\
\hline Nausea & 8 (21.6) & $0(0.0)$ \\
\hline Leukopenia & 7 (18.9) & $6(16.2)$ \\
\hline Hypertension & $5(13.5)$ & $2(5.4)$ \\
\hline Vomiting & $5(13.5)$ & $0(0.0)$ \\
\hline $\begin{array}{l}\text { Aspartate aminotransferase } \\
\text { increased }\end{array}$ & $5(13.5)$ & $0(0.0)$ \\
\hline C-reactive protein increased & $5(13.5)$ & $0(0.0)$ \\
\hline $\begin{array}{l}\text { Alanine aminotransferase } \\
\text { increased }\end{array}$ & $4(10.8)$ & $1(2.7)$ \\
\hline Hypotension & $4(10.8)$ & $1(2.7)$ \\
\hline Asthenia & $4(10.8)$ & $0(0.0)$ \\
\hline Hyperhidrosis & $4(10.8)$ & $0(0.0)$ \\
\hline
\end{tabular}

$A E$ adverse event, $S A E$ serious adverse event

Thirty-five patients (94.6\%) had $\geq 1$ treatment-related AE (Table 1); the most frequent were infusion-related reactions (IRRs), chills, and pyrexia. The most frequent treatmentrelated grade 3/4 AEs were neutropenia, thrombocytopenia, anemia, and leukopenia. Twelve patients (32.4\%) had treatment-related serious AEs, most frequently neutropenia (8.1\%), IRRs, pyrexia, and leukopenia (all 5.4\%). There were no cases of drug-related tumor lysis syndrome. Ten patients (27.0\%) had AEs that resulted in discontinuation of BI 836826. AEs that most frequently resulted in discontinuation were thrombocytopenia and IRRs (both 5.4\%). There were no fatal on-treatment AEs. IRRs were observed at all dose levels, most frequently in cycle 1 (Suppl. Fig. 2). IRRs generally occurred during infusion and quickly responded to standard management including treatment interruption and symptomatic therapy. IRRs decreased in frequency and severity following introduction of a modified, stepwise, infusion scheme (Supplementary Patients and Methods). 


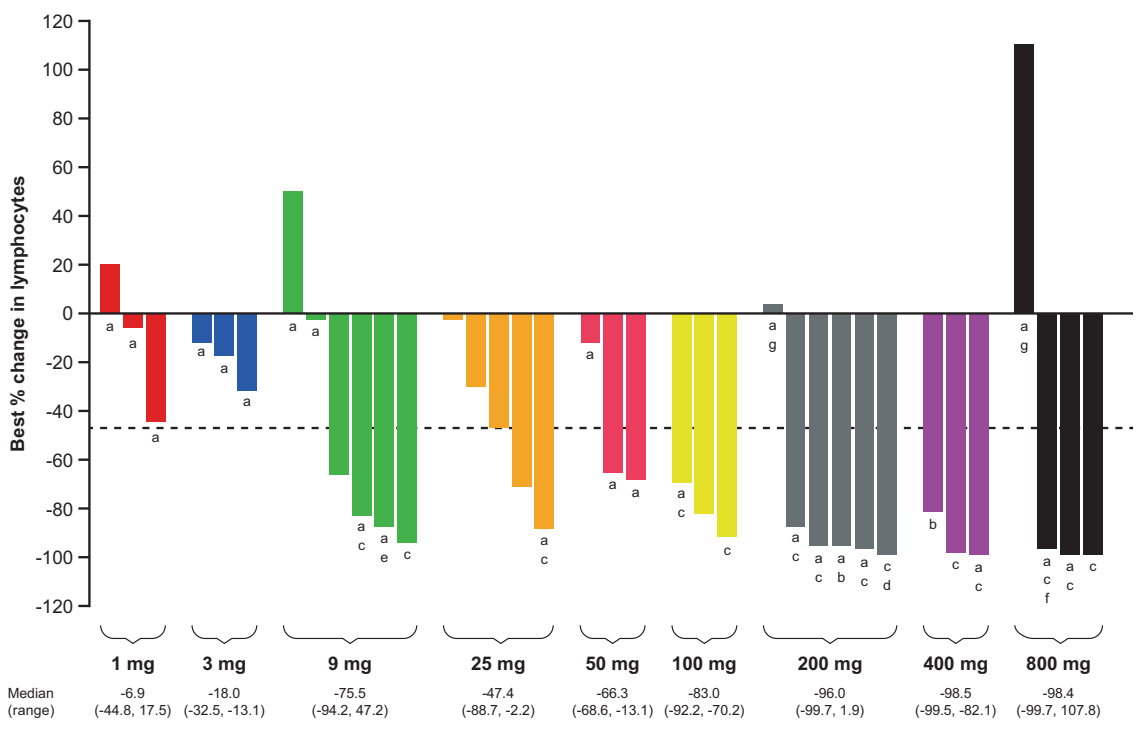

Fig. 1 Best percentage change from baseline in peripheral blood lymphocyte count by patient. a Patients presented with del(17p) and/or TP53 mutation. b Patients had a baseline lymphocyte count of $<4 \times$ $10^{9} / \mathrm{L}$. c Patients had a best percentage change from baseline to a lymphocyte count of $<4 \times 10^{9} / \mathrm{L}$. d Patient was randomized to $200 \mathrm{mg}$ but was escalated to $400 \mathrm{mg}$. e Patient was randomized to $9 \mathrm{mg}$ but

Baseline neutrophil, hemoglobin, and platelet counts were low in $21.6 \%, 35.1 \%$, and $51.4 \%$ of patients, respectively (Table 1). During treatment, at least one neutrophil, hemoglobin, or platelet measurement declined to $<0.5 \times 10^{9} / \mathrm{L}$ (grade 4), $<80 \mathrm{~g} / \mathrm{L}$ (grade 3 ), or $<25 \times 10^{9} / \mathrm{L}$ (grade 4 ) in $62.1 \%, 43.2 \%$, or $29.7 \%$ of patients, respectively. The degree of preexisting cytopenia in these patients is shown in Suppl. Fig. 3. Neutrophil and platelet counts declined immediately after the end of the infusion, with recovery prior to the next dose in most patients (Suppl. Fig. 4). Fifteen (40.5\%) patients had grade 4 neutropenia lasting $\geq 7$ days; none had concomitant CTCAE grade $\geq 3$ infections. Five patients $(13.5 \%)$ had grade 4 thrombocytopenia lasting $\geq 7$ days; none had concomitant bleeding events of CTCAE grade $\geq 3$.

Clinically relevant reductions in lymphocyte count were observed in all dose cohorts $\geq 9 \mathrm{mg}$ (Fig. 1), including patients with a del(17p) or TP53 mutation. Improvements in hemoglobin, neutrophil, and platelet counts are summarized in the Supplementary results. Thirteen patients had an objective response (35.1\%; all PRs). At doses $\geq 9 \mathrm{mg}$ and $\geq 200 \mathrm{mg}$, objective response rates (ORRs) were $41.9 \%$ (13/31 patients) and $61.5 \%$ (8/13 patients), respectively. ORR in patients with a del(17p) mutation and/or a TP53 mutation was $45.8 \%$ $(11 / 24$ patients). ORR in rituximab-refractory patients was $47.6 \%$ (10/21 patients). All evaluable patients achieved disease control (complete response, PR, or stable disease).

Plasma exposure was highly variable and increased more than dose proportionally (Suppl. Table 3). No accumulation of plasma concentrations was observed after repeated was escalated to $25 \mathrm{mg}$. f Patient was randomized to $800 \mathrm{mg}$ but was de-escalated to $600 \mathrm{mg}$ in cycle 3 and received $800 \mathrm{mg}$ again by error in cycle 4. $g$ Patients received only the predose, i.e., the first part of the split infusion of BI 836826 in cycle 1, which was $10 \%$ of the total dose, but maximally $10 \mathrm{mg}$

administration. Following the end of infusions, plasma concentrations decreased rapidly and were below the limit of quantification by $336 \mathrm{~h}$ for doses $<400 \mathrm{mg}$. Plasma clearance decreased with increasing doses, suggesting target-mediated drug clearance. Also, the volume of distribution decreased with increasing doses, with moderate variability across all dose groups.

At screening, CD37 expression on B cells was observed in all 35 evaluable patients. The median percentage of CD19+/CD37+ B cells was 86.4 (range 12.7-95.3; standard deviation: 21.3). A change in peripheral CD19+ B cells was seen at $\geq 9 \mathrm{mg}$, with a reduction following the first infusion. A clinically relevant and sustained reduction became evident from doses approximately $\geq 100 \mathrm{mg}$ (Suppl. Fig. 5).

These data represent the first human experience with BI 836826, and indicate that it is tolerable up to at least 400 $\mathrm{mg}$; additional doses of 400-800 mg remain unexplored due to study termination. The AE profile was similar to other Fc-modified antibodies [6-8], consisting predominantly of IRRs, neutropenia, and thrombocytopenia, which were manageable with supportive care. The frequency of IRRs and those resulting in treatment discontinuation were similar to those observed with other antibodies such as obinutuzumab and XmAb5574 in patients with CLL and were observed despite premedication [7, 8]. IRRs with BI 836826 are not considered a barrier to further development, and may be reduced by combination strategies that debulk CLL 
burden prior to initial infusion. Neutropenia and thrombocytopenia were not associated with severe infections or bleeding. Their rapid kinetics and recovery suggest that cytopenia may be related to direct effects of BI 836826 on mature peripheral blood cells, as supported by preclinical data (unpublished). Prolonged administration of BI 836826 was possible without cumulative toxicity, with two patients receiving almost 3 years of treatment.

BI 836826 demonstrated notable clinical activity, achieving a clearance of CLL cells from peripheral blood. At doses that showed the strongest pharmacodynamic effect on B-lymphocyte counts, the ORR was $61.5 \%$. Responses were also observed in patients with $\operatorname{del}(17 \mathrm{p})$ or TP53 mutations. Although phase 1/2 studies are limited by small patient numbers and dose variations, response rates with BI 836826 compare favorably to otlertuzumab or rituximab as single agents in CLL $[9,10]$, and are in the range observed with ofatumumab and obinutuzumab [6, 11]. Moreover, there is preclinical rationale to use BI 836826 in combination with cytotoxics and nonchemotherapy agents such as idelalisib [12, 13].

Overall, this study indicates that BI 836826 treatment in patients with CLL is a valid approach with acceptable tolerability and notable efficacy, especially in difficult-to-treat patients with poor-risk features including del(17p) or TP53 mutations. CD37 is a promising therapeutic target that warrants further clinical investigation in CLL.

Acknowledgements We thank the patients, their families and all of the investigators who participated in these studies. The conduct of this research, study design, data collection, and analysis was financially supported by Boehringer Ingelheim. Medical writing assistance, supported financially by Boehringer Ingelheim, was provided by Lynn Pritchard, DPhil, of GeoMed, an Ashfield company, part of UDG Healthcare plc, during the preparation of this manuscript. The authors were fully responsible for all content and editorial decisions, were involved at all stages of manuscript development and have approved the final version.

Author contributions UUK managed study, revised and approved the final manuscript. SS performed research and collected and interpreted data, revised and approved the final manuscript. TAS, FO, J-FR, WS, FL, BE, LY, and TZ performed research and collected data, revised and approved the final manuscript. PB designed research, interpreted data, wrote, revised and approved the final manuscript. UVW analyzed data, revised and approved the final manuscript.

\section{Compliance with ethical standards}

Conflict of interest SS reports consultancy, speaker honoraria and research grants from AbbVie, Amgen, AstraZeneca, Boehringer Ingelheim, Celgene, Gilead, GSK, Hoffmann La-Roche, Janssen, and Novartis. BE reports consultancy for Gilead, Roche, Janssen, Abbvie, Novartis, Celgene, and research funding from Gilead, Roche, Janssen, Abbvie, and honoraria from Gilead, Roche, Janssen, Abbvie, Novartis, Celgene. UVW, UUK, and PB report employment for Boehringer Ingelheim Pharma GmbH \& Co KG. FL reports consultancy, research funding, honoraria and payment for expert testimony from Novartis,
Incyte, and BMS. TZ holds a patent (WO2012007576A1). The remaining authors have nothing to disclose.

Publisher's note: Springer Nature remains neutral with regard to jurisdictional claims in published maps and institutional affiliations.

Open Access This article is licensed under a Creative Commons Attribution 4.0 International License, which permits use, sharing, adaptation, distribution and reproduction in any medium or format, as long as you give appropriate credit to the original author(s) and the source, provide a link to the Creative Commons license, and indicate if changes were made. The images or other third party material in this article are included in the article's Creative Commons license, unless indicated otherwise in a credit line to the material. If material is not included in the article's Creative Commons license and your intended use is not permitted by statutory regulation or exceeds the permitted use, you will need to obtain permission directly from the copyright holder. To view a copy of this license, visit http://creativecommons. org/licenses/by/4.0/.

\section{References}

1. Gomes LC, Ferrao ALM, Evangelista FCG, de Almeida TD, Barbosa RC, Carvalho MDG, et al. Advances in chronic lymphocytic leukemia pharmacotherapy. Biomed Pharmacother. 2018;97:349-58.

2. Bertoni F, Stathis A. Staining the target: CD37 expression in lymphomas. Blood. 2016;128:3022-3.

3. Lapalombella R, Yeh YY, Wang L, Ramanunni A, Rafiq S, Jha S, et al. Tetraspanin CD37 directly mediates transduction of survival and apoptotic signals. Cancer Cell. 2012;21:694-708.

4. Heider KH, Kiefer K, Zenz T, Volden M, Stilgenbauer S, Ostermann E, et al. A novel Fc-engineered monoclonal antibody to CD37 with enhanced ADCC and high proapoptotic activity for treatment of B-cell malignancies. Blood. 2011;118:4159-68.

5. Hallek M, Cheson BD, Catovsky D, Caligaris-Cappio F, Dighiero $\mathrm{G}$, Dohner H, et al. Guidelines for the diagnosis and treatment of chronic lymphocytic leukemia: a report from the International Workshop on Chronic Lymphocytic Leukemia updating the National Cancer Institute-Working Group 1996 guidelines. Blood. 2008;111:5446-56.

6. Cartron G, de Guibert S, Dilhuydy MS, Morschhauser F, Leblond V, Dupuis J, et al. Obinutuzumab (GA101) in relapsed/refractory chronic lymphocytic leukemia: final data from the phase $1 / 2$ GAUGUIN study. Blood. 2014;124:2196-202.

7. Goede V, Fischer K, Busch R, Engelke A, Eichhorst B, Wendtner $\mathrm{CM}$, et al. Obinutuzumab plus chlorambucil in patients with CLL and coexisting conditions. N Engl J Med. 2014;370:1101-10.

8. Woyach JA, Awan F, Flinn IW, Berdeja JG, Wiley E, Mansoor S, et al. A phase 1 trial of the Fc-engineered CD19 antibody XmAb5574 (MOR00208) demonstrates safety and preliminary efficacy in relapsed CLL. Blood. 2014;124:3553-60.

9. Huhn D, von Schilling C, Wilhelm M, Ho AD, Hallek M, Kuse R, et al. Rituximab therapy of patients with B-cell chronic lymphocytic leukemia. Blood. 2001;98:1326-31.

10. Byrd JC, Pagel JM, Awan FT, Forero A, Flinn IW, DeaunaLimayo DP, et al. A phase 1 study evaluating the safety and tolerability of otlertuzumab, an anti-CD37 mono-specific ADAPTIR therapeutic protein in chronic lymphocytic leukemia. Blood. 2014;123:1302-8.

11. Wierda WG, Kipps TJ, Mayer J, Stilgenbauer S, Williams CD, Hellmann A, et al. Ofatumumab as single-agent CD20 immunotherapy in fludarabine-refractory chronic lymphocytic leukemia. J Clin Oncol. 2010;28:1749-55. 
12. Betrian S, Ysebaert L, Heider KH, Delord JP, Fournie JJ, QuilletMary A. Idelalisib improves CD37 antibody BI 836826 cytotoxicity against chemo-resistant/relapse-initiating CLL cells: a rationale for combination treatment. Blood. Cancer J. 2016;6: e496.
13. Krause G, Baki I, Kerwien S, Knodgen E, Neumann L, Gockeritz E, et al. Cytotoxicity of the CD37 antibody BI 836826 against chronic lymphocytic leukaemia cells in combination with chemotherapeutic agents or PI3K inhibitors. Br J Haematol. 2016; 173:791-4.

Leukemia (2019) 33:2535-2539

https://doi.org/10.1038/s41375-019-0483-z

Minimal resdiual disease

\title{
getITD for FLT3-ITD-based MRD monitoring in AML
}

\author{
Tamara J. Blätte ${ }^{1,2} \cdot$ Laura K. Schmalbrock $^{1} \cdot$ Sabrina Skambraks $^{1} \cdot$ Susanne Lux $^{1} \cdot$ Sibylle Cocciardi $^{1}$ • \\ Anna Dolnik ${ }^{1,2} \cdot$ Hartmut Döhner $^{1} \cdot$ Konstanze Döhner $^{1} \cdot$ Lars Bullinger $^{2}$
}

Received: 29 January 2019 / Revised: 20 March 2019 / Accepted: 22 March 2019 / Published online: 14 May 2019

(c) The Author(s) 2019. This article is published with open access

\section{To the Editor:}

The clinical relevance of measurable residual disease (MRD) monitoring has been well recognized in acute myeloid leukemia (AML) [1] and respective assays have been established for several recurrent leukemic markers $[2,3]$. However, although internal tandem duplications in the FLT3 gene (FLT3-ITDs) are the most common poor prognosis AML drivers [4], they have remained a challenging target: Their heterogeneity makes conventional PCRbased methods either insensitive or laborious [5]. Yet with recently approved FLT3-kinase inhibitors available [6], a specific monitoring of FLT3-mutation loads, and thus response to targeted therapy, is of particular interest. Nextgeneration sequencing (NGS) workflows for FLT3-ITD monitoring have been described, but were previously either proprietary or undisclosed $[7,8]$, unable to detect and correctly annotate all of the tested ITDs $[9,10]$, or used in

These authors contributed equally: Konstanze Döhner, Lars Bullinger

Supplementary information The online version of this article (https:// doi.org/10.1038/s41375-019-0483-z) contains supplementary material, which is available to authorized users.

Lars Bullinger

lars.bullinger@charite.de

1 Department of Internal Medicine III, University Hospital of Ulm, Ulm, Germany

2 Department of Hematology, Oncology and Tumor Immunology, Charité University Medicine, Berlin, Germany conjunction with manual analysis with inherently subjective results [10].

We have therefore developed a new method based on targeted high-coverage NGS and our novel, opensource analysis program getITD. For assay assessment, we sequenced 3 human AML cell lines (Leibniz Institute DSMZ-German Collection of Microorganisms and Cell Cultures, Braunschweig, Germany), 2 healthy volunteers, and 57 samples from 28 AML patients who were all included in the AMLSG BiO Registry study (NCT01252485) and gave their informed consent according to the Declaration of Helsinki. We show that our workflow detects ITDs of a broad range of lengths, insertion sites and variant allele frequencies (VAFs) with high accuracy and precision, is fully objective without any requirement for manual analysis, and thus applicable to routine clinical monitoring. Sample and method details are provided as supplementary information. getITD is freely available at https://github.com/tjblaette/getitd.

To demonstrate our assay's specificity, we analyzed three FLT3-ITD negative control samples (peripheral blood of healthy volunteers, $n=2$; AML cell line HL-60). No ITDs were reported, indicating an assay specificity of $100 \%$ (coverage: 1.1-4.2 million, mean 2.6 million paired-end reads). To assess sensitivity, we analyzed two serial dilutions of FLT3-ITD positive in FLT3-ITD-negative DNA from AML cell lines MOLM-14 (21 bp ITD, 67\% VAF [11]), PL-21 (126 bp ITD, 33\% VAF [11]), and HL-60 (FLT3-ITD negative [11]). For each of the ITD-positive cell lines, we sequenced undiluted DNA and 3-4 serial 1:10 dilutions in HL-60 (1.1-2.9 million, mean 2.0 million paired-end reads). The expected ITDs were detected in all samples and VAF estimates were accurate and decreased 